\title{
A Dynamic Optimization for Automotive Vehicle Shipment and Delivery
}

\author{
John Yee* \\ 자동차 선적 및 납기를 위한 동적 최적화
}

John Yee*

\section{ABSTRACT}

The automotive industry has made much efforts to deliver finished vehicles to customers with speed and reliability. Decreasing the time a vehicle stays within an assembly plant from production release to shipment contributes to reduce the total order lead-time and consequently, the total transportation cost as well. Conventional shipment planning algorithms are limited in accommodating the dynamics of assembly plant operations as to finished vehicle shipment. This paper presents a market-based multi-agent shipment planning algorithm to optimize the performance of vehicle shipment process, capturing the operationally disruptive events. Experimental results using simulation show that the algorithm improves vehicle shipment performance with respect to lead time, labor efficiency, finished product quality, and transportation efficiency.

Key words : vehicle shipment, multi-agent, supply chain management, dynamic optimization, market-based

\section{요약}

완성차의 빠르고 안전한 납기를 위해 자동차 업계는 많은 노력을 기울여 왔다. 생산 후 선적까지 완성차가 조립공장내에 체재하는 시간의 감축을 통해 총 주문시간을 줄일 수 있음과 동시에 총수송비용 또한 줄일 수 있다. 전통적인 선적계획법들은 조립공장내에서 발생하는 동적인 사건들을 다루는데 한계가 있다. 본 논문은 이러한 선적과정중에 발생할 수 있는 동적 사건들 을 해결하는 다중 에이전트 기반 선적 방법을 제시하고 있다. 시뮬레이션을 이용한 실험 결과를 통해 이러한 방법이 주문시간, 작업효율, 품질 및 수송효율의 증진을 가져올 수 있음을 보였다.

주요어 : 자동차 선적, 다중 에이전트, 공급망관리, 동적 최적화, 시장 기반

\section{Introduction}

The automotive companies have been executing a business model, say, the order-to-delivery (OTD), to achieve order lead time reduction, order delivery date reliability improvement, and order visibility to customers. The vision of the OTD is defined ${ }^{[1]}$ as "Personalized vehicles provided with zero customer inconvenience".

Received: 14 November 2014, Revised: 27 November 2014, Accepted: 16 December 2014

*Corresponding Author: John Yee

E-mail: styee@hotmail.com

U.S. Department of the Treasury, Sungkyunkwan University
Its challenge is to transform the automotive industry to a sense-and-respond enterprise focused on customers. This transformation impacts business operations of every supply chain member: suppliers, manufacturers, distributers, and dealers, and it requires changes in their business processes. The key objectives of the OTD business model are as follows:

(1) Order-to-delivery lead-time reduction between dealer/customer order and vehicle delivery.

(2) Order delivery date reliability improvement to provide dealer/customer with reliable delivery date at the time of order placement.

(3) Real-time customer experience by providing 
web-enabled resources for supply chain visibility.

(4) Supply chain constraints elimination to quickly sense and meet true market demand.

An automaker wants to respond to customer's personalized vehicle request through fast, flexible, and interactive business processes across the extended enterprise. The automaker's commitment for satisfying the customer's choice of vehicle, delivery time, and delivery location enhances customer confidence. In the OTD business model, customer demand triggers an order for raw material, production, and subsequent shipment of the finished vehicle to the delivery location, typically, a dealer. The customer demand drives the production schedule and material procurement as well as the scope and size of resources and the capacity of supply, production, and logistics processes.

Finished vehicles are moved from assembly plant to its adjacent shipping yard and held until ready for shipment to dealers or distribution centers. An automotive company in the U.S. has 20 to 30 assembly plants and 30 to 40 vehicle distribution centers in North America where the vehicles reside for a few days for shipment mix and consolidation. The amount of time each vehicle stays at these facilities constitutes a big portion of the total OTD time, and decreasing this time is crucial to achieve the OTD lead-time reduction. In addition, if the automaker wants to ensure the visibility of all the vehicles along the delivery chain, it can provide the customers with more reliable delivery dates as well as better order status. For each objective stated above, the automaker should investigate a solution. Whereas the automaker should invest in a new technology to achieve the last two objectives, the first two objectives could be accomplished in part by improving the current vehicle shipment practices. Vehicle shipment environment consists in inherent dynamic nature. Conventional shipment methods are limited to handle daily occurring disruptive events. This paper is to present a new method for dynamically optimizing vehicle shipment process by taking all the dynamic events into account for building shipment loads.

This paper is organized as follows. Next section presents the methodology that accommodates the dynamic shipment method, while stating the limitations of conventional shipment methods. Then, analysis results are provided. Next, managerial implications are discussed. Finally, conclusions are provided.

\section{Methodology}

\subsection{Vehicle Shipment Environment}

Every day, thousands of vehicles are produced at an automotive assembly plant. As shown in Fig. 1, once a vehicle is completely assembled, it is inspected and transferred to yard operator at hand-off area, and yard operator deploys the vehicle in an empty parking spot. The vehicles deployed in yard are shipped out to dealers or distribution centers via two modes of transportation, namely, truck and rail. The deployment is an activity to put a vehicle in a particular bay of the yard and the shipment is about building shipment loads for delivering the vehicles. If the vehicle is transported by rail, it is deployed in rail shipment parking area. If the vehicle is transported by truck, it is deployed in truck shipment parking area. A certain portion of the vehicles deployed at the plant yard is put on hold for various reasons: bad parts from suppliers, production quality defects, carrier unavailability, and insufficient dealer lot to hold vehicles. When the plant quality department needs to locate a range of vehicles to put on hold, it is time consuming and labor intensive. Despite that, it is critical to locate the vehicles having some deviations in the plant before shipping because more efforts are needed to resolve the deviations at dealer sites. A vehicle could be deployed in a wrong spot, called misbay (several times a day),

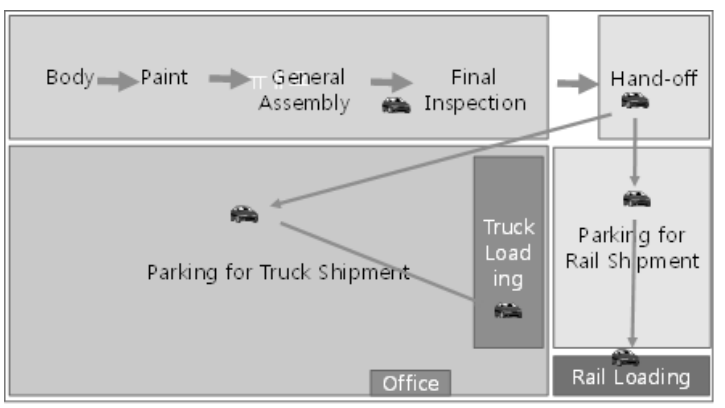

Fig. 1. Vehicle shipment environment 
and loaded in a wrong truck/rail, called misload (several times a week). If either a misbay or a misload is found before shipment, a couple of hours is needed for correction. If this problem is discovered after the shipment, it costs much more, say, three to four times more. These operational disruptions could occur at the shipment yard anytime, and cause prolonged vehicle dwell time (the time a vehicle stays at a facility, e.g., here at the yard), shipment abnormality, and labor cost increase.

A distribution center has similar operational environment and, additionally, it sorts out the vehicles shipped from different assembly plants by geographical regions and consolidates the vehicles going to similar destinations. The distribution centers should maintain high customer service level by efficient delivery schedule to meet the available-to-promise (ATP) commitments. The ATP is the promised delivery date issued by an automaker to the customer and is one of the important performance measures in the automotive industry to determine the customer service level which offers a competitive advantage in the market.

\subsection{Conventional Shipment Methods}

The shipment decision problem, also called load makeup problem, is to load a set of vehicles with different destinations onto either trucks or trains to minimize both total vehicle dwell time and total transportation cost. The load makeup planning is a two-layer NP-hard problem with two conflicting objectives. That is, the yard manager wants to minimize the total transportation distance as well as to ship out the vehicles that are stayed longest first. Three algorithms are applicable to build the shipment loads in a static manner: Empirical (EM), Minimum Spanning Tree (MST), and Vehicle Routing Optimization (VRO).

The EM algorithm imitates the planning behavior of the yard manager. Every morning the yard manager obtains both shipment truck and train schedules for the same day and current vehicle inventory information of the yard. The yard manager finds an oldest vehicle that will become the leading vehicle in load building. Starting from the leading vehicle, the manager starts searching for the vehicles having closer destinations to the leading one. The vehicles going to the same dealer of the leading vehicle are selected first and next, the vehicles having the destinations of the same demand area, typically, adjacent cities or states, are chosen until the capacity of a truck or a train is filled out. However, the yard manager does not consider uncertain dynamics that could occur during the day.

In the MST algorithm, instead of using the concept of demand areas and their adjacency relationships, each load clusters its vehicles using the rule of minimum spanning tree, which is usually used to find a tree in a graph with the minimum summation of weights of connecting $\operatorname{arcs}^{[2]}$. The distance between any two dealers is already known. Different from normal MST, the MST for load makeup is restricted such that the size of each tree is limited to the capacity of the corresponding truck. The algorithm starts from an oldest or a customized order vehicle. In the searching process, the whole set of vehicles is divided into two exclusive sets: one set contains the vehicles already assigned to loads and the other contains the vehicles not assigned to loads. A priority queue stores all the pairs of vehicles with the enumeration of all the loaded vehicles and all the unloaded vehicles. The queue is sorted out by the distance of two vehicles in a pair. During each step, the pairs are composed of the vehicle just added into the loaded set and all the other vehicles are inserted into the priority queue. Then, the top pair with minimum distance is chosen and the relevant vehicle is moved from the unloaded set to the loaded set, with other constraints checked. Finally, the pairs related to the chosen vehicle are all deleted from the queue. After all the loads are built, a traveling salesman problem (TSP) is solved for each load. The MST algorithm is an approximate approach because the TSP is not directly used for clustering. Refer to Kim and et al. ${ }^{[3]}$ for more details.

The VRO algorithm for the load makeup has two distinctions compared with normal vehicle routing problem: first, the capacities of trucks are different; second, there are more vehicles available than scheduled number of trucks. The VRO algorithm is a composite method that consists of two main steps. In the first 


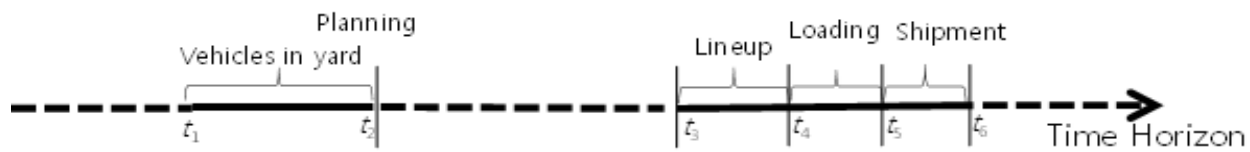

Fig. 2. Static shipment process

step, multiple routes are constructed sequentially for all the available trucks by assuming enough trucks are available for load makeup. In the second step, the routes constructed are improved based on heuristic rules that exchange some nodes between two routes.

\subsection{Limitations of Conventional Shipment Algorithms}

Although the MST and VRO algorithm could improve the load makeup performance to a certain extent, they are not good enough like the EM algorithm to take into account the most recently updated information regarding both the vehicles and the yard. Between a load building and a truck arrival, yard conditions may change and unexpected events may occur. These uncertainties could disrupt the entire shipment plan. As shown in Fig. 2, vehicles are deployed at the yard from $t_{1}$ to $t_{2}$. At $t_{2}$, loads are built only for the vehicles physically stayed in the yard. From $t_{3}$ to $t_{4}$, when trucks arrive at the yard, the vehicles are lined up for loading. The vehicles are loaded on the trucks from $t_{4}$ to $t_{5}$ and shipped out from $t_{5}$ to $t_{6}$ in a given sequence. In other words, load makeup is being done in a structured, sequential manner based on the vehicle information available at $t_{2}$. However, during any time between $t_{1}$ to $t_{4}$ (even for the time from $t_{4}$ to $t_{5}$ ), the yard and vehicle status could be dynamically changed according to various regular and exceptional events, and these changes should be captured in load planning and lineup process. Dynamically occurring events include the new vehicle arrivals, the vehicles redeployed from quality holds and repairs, the vehicles changed in bay locations, and the schedule changes of trucks assigned to the yard. In addition, load building should consider other information, such as, advanced production notifications, projected truck dispatching schedules, predicted future shipment schedules, and yard per- sonnel availability status. More importantly, these uncertain disruptive events need to be captured in the shipment algorithm for optimal decision-making. It is very challenging, perhaps, impossible, for both the MST and the VRO algorithm to model these dynamic behavior.

\subsection{Dynamic Optimization}

We may have two strategies for load makeup planning. One strategy is to just use the EM, MST, or VRO algorithm for building every load based on static environment information. This strategy can be prone to expensive computation, which requires a large amount of computation time that may be longer than the time gap between load makeups and thus, might not produce the resulting loads within a given time. From operational perspectives, solving the whole problem at every load building could totally change the previous solution, which causes inconsistency for shipment process, that is, too much change could occur in vehicle-destination pairs of the resulting loads. Also, this strategy is not effective to capture the difficult-to-model dynamic information mentioned above.

The other strategy is to search the optimal solution starting from an existing solution. This strategy is also termed as dynamic optimization or continual optimization ${ }^{[4]}$ that was adopted in this paper. Instead of solving the entire problem again, a new solution is sought from the solution obtained by one of the static algorithms. This strategy may avoid three drawbacks of the first strategy: high computational time, solution inconsistency, and information modeling ineffectiveness. The concept of dynamic optimization is in some sense similar to postoptimality analysis of linear programming using simplex method, in which the simplex pivots start with an existing optimal solution before the parameters or constraints are changed ${ }^{[5]}$. 
For our dynamic optimization of the load makeup, we need to do the load building anytime on demand according to the yard status, and the solution obtained from a static optimization is used as an initial solution. This makes sense practically because the possible number of load makeups cannot be infinite because the number of shipment trucks and vehicles is finite. Different from static optimization, any dynamic changes occurring between load planning periods are tracked and used to trigger incremental optimization.

\subsubsection{Market-based Multi-agent System Architecture}

To achieve the dynamic optimization, we need a computational architecture and a mechanism to effectively collect and coordinate the dynamical events among several operational players of the yard. A multi-agent system (MAS) has been suggested as a promising information infrastructure and solution approach for planning problems that are distributed, complex, and heterogeneous ${ }^{[6,7]}$. A leader-follower problem was discussed for a multi-agent system having a switching interconnection topology ${ }^{[8]}$. A distributed computation model was studied to optimize convex objective functions of multi-agent system using subgradient optimization ${ }^{[9]}$. A constrained consensus and optimization was presented for multi-agent networks with time-varying connectivity ${ }^{[10]}$. Agent-based approaches and classic optimization techniques, including heuristic search, are compared for dynamically distributed resource allocation ${ }^{[11]}$. Agent-based approaches fit better to situations where problem domain is large, node or link failure is high, frequency of decision-making is high, problem structure is frequently changed, and private or local information is important. Our problem exhibits all these characteristics that make MAS a good choice for our purpose. Because the shipment yard involves multiple parties and has the characteristics of a market, we can establish the MAS framework by defining a number of agents to which we assign different functions of the market. In addition, the concept of market equilibrium is analogous to the incremental optimality that can change the optimal solution according to the dynamics influencing the shipment process. From the perspective of game theory, the equilibrium of a perfect market is reached at the point where no one is interested in selling and buying. This concept is used to decide when the market heuristic process should stop $^{[12]}$. As previous research, the author presented an agent-based shipment algorithm for capacitated vehicle routing problem with load balancing ${ }^{[13]}$.

Three types of agents are created: yard agent, load agents, monitor agent. The yard agent represents the shipment yard. Its behavior includes: monitoring and updating vehicle location database to detect newly available vehicles; deriving dwell time for a vehicle and lineup time for a load; having future production schedules; serving as a trading agent in the market heuristic. A load agent represents a truck and its main behavior includes: building a load using the guaranteed vehicle information; monitoring lineup and execution of the load; serving as a trading agent in the market heuristic. The monitor agent monitors and coordinates buying and selling proposals and decides winning agents.

To emulate a market in the shipment environment, a yard agent and a group of load agents are designated as traders, vehicles as products, and cost savings as profits. The yard agent and the load agents propose their selling or buying proposals based on their constraints, capabilities, and interests. The market monitor collects these proposals, and each of which is identified with a vehicle. The yard agent, multiple load agents, and the market monitor constitute all the players in the emulated market.

In normal trading, double auction interactions take place, that is, a buyer has the ability to buy a vehicle from anybody who wants to sell. One round of interaction has two proposing steps. In the first step, the selling agents propose their asks; in the following step, the buying agents make their bids based on the asks proposed in the first step. With the collected proposals, the monitor agent then decide the winners of double auction.

\subsubsection{Sell/Buy Decisions}

The selling decision problem is to determine which vehicle a load agent or the yard agent sells. First, consider the sell decision of a load agent. Let $I_{j}$ denote 
the set of destinations that truck $j$ ( $j=0$ for the yard agent) plans to deliver the corresponding vehicles. The decision problem is to determine the vehicle with destination $\alpha_{j}^{*}\left(\alpha_{j}^{*} \in I_{j}\right)$ to be deleted. The vehicle to be deleted is proposed for selling. A deterministic decision is made through finding the vehicle with the maximum deletion cost and is described in Equation (1). $\Delta c_{j}^{-}\left(\alpha_{j}\right)$ represents the deletion cost of removing the vehicle with destination from the current load agent that maintains minimum total distance for all the possible links that truck travels.

$$
\alpha_{j}^{*}=\underset{\alpha_{j} \in I_{j}}{\operatorname{argmax}} \Delta c_{j}^{-}\left(\alpha_{j}\right)
$$

Second, when the yard agent sells a vehicle, the deterministic decision can be made by

$$
\alpha_{0}^{*}=\underset{\alpha_{0} \in I_{0}}{\arg \max } \Delta c_{j}^{-}\left(\alpha^{*}\right)-\Delta c_{j}^{+}\left(\alpha_{0}\right) .
$$

$\Delta c_{j}^{+}\left(\alpha_{0}\right)$ denotes the insertion cost of adding the vehicle with destination $\alpha_{0}$ from the yard agent to the load agent. The stochastic decision is based on the probability distribution related to the deletion cost of all the vehicles in the load agent using Equation (2). Likewise, the stochastic decision is made for the yard agent accordingly.

$$
\begin{aligned}
& P_{j}^{\text {sell }}\left(\alpha_{j}\right)=\frac{\Delta c_{j}^{-}\left(\alpha_{j}\right)}{\sum_{\gamma_{j} \in I_{j}} \Delta c_{j}^{-}\left(\gamma_{j}\right)}, \forall \alpha_{j} \in I_{j} \\
& P_{0}^{\text {sell }}\left(\alpha_{0}\right)=\frac{\triangle c_{j}^{-}\left(\alpha_{j}^{*}\right)-\Delta c_{j}^{+}\left(\alpha_{0}\right)}{\sum_{\gamma_{j} \in I_{0}} \Delta c_{j}^{-}\left(\gamma_{j}^{*}\right)-\triangle c_{j}^{+}\left(\gamma_{0}\right)}, \forall \alpha_{0} \in I_{0}
\end{aligned}
$$

The buying decision problem is to determine which vehicle a load agent buys. Let $I^{\text {sell }}$ denote the set of destinations whose corresponding vehicles are proposed to be sold by all the selling agents during one round of double auction interaction. The decision problem is for the load agent $j$ to determine the vehicle with destination $\beta_{j}^{*} \in I^{\text {sell }}$ to be included in its routing plan. The deterministic decision is to find the destination with the maximum positive benefit, which is described in Equation (3), where the dot means that every selling agent can be considered. When the load agent $j$ buys $\beta_{j}$, choose the selling agent that results in maximum positive benefit globally. This means we should consider the deletion cost of $\beta_{j}$ from the selling agent satisfying Equation (3). The yard agent has no right to buy.

$$
\beta_{j}^{*}=\underset{\beta_{j} \in I^{\text {sell }}}{\arg \max }\left|\Delta c_{j}^{+}\left(\beta_{j}\right)-\Delta c_{.}^{-}\left(\beta_{j}\right)\right|_{\Delta c_{j}^{+}\left(\beta_{j}\right)-\Delta c_{.}^{-}\left(\beta_{j}\right)>0}
$$

Likewise, the probability distribution in the stochastic model can be calculated by Equation (4).

$$
P_{j}^{\text {buy }}\left(\beta_{j}\right)=\frac{\left|\Delta c_{j}^{+}\left(\beta_{j}\right)-\Delta c^{-}\left(\beta_{j}\right)\right|_{\Delta c_{j}^{+}\left(\beta_{j}\right)-\Delta c_{c}^{-}\left(\beta_{j}\right)>0}}{\sum_{\gamma_{j} \in \Gamma^{r \text { ell }}}\left|\Delta c_{j}^{+}\left(\gamma_{j}\right)-\Delta c^{-}\left(\gamma_{j}\right)\right|_{\Delta c_{j}^{c}\left(\gamma_{j}\right)-\Delta c_{.}^{-}\left(\gamma_{j}\right)>0}}, \forall \beta_{j} \in I^{\text {sell }}
$$

A special interaction is designed to handle the situation of fully capacitated load agents. At the beginning of dynamic optimization, most load agents are full with vehicles and have no ability to buy any other vehicles. We design surplus trading to deal with this situation. In the surplus trading, a fully capacitated load agent exchanges one of its vehicles with one of the vehicles of the yard agent. Since a fully capacitated load agent has no vacancy to include another vehicle in its routing plan, it is only interested in trading in a vehicle from the yard agent and trading out one of its own vehicles. The vehicle to trade out is called a surplus vehicle, which is left over and to be returned to the yard agent. The surplus trading between a fully capacitated load agent and the yard agent keeps the balance of the number of vehicles between the shipment yard and the scheduled trucks. The balance is one of the important constraints of the original load makeup planning problem. In the surplus trading, the yard agent 
becomes passive and does not actively make decisions, but provides the vehicle requested by the load agent and always accepts the surplus vehicles without conditions. The decision-making in the surplus trading can be deterministic or stochastic. The deterministic model addresses the self-interest of the load agent. The stochastic model makes the load agent possibly yield its self-interest to other load agents.

\subsubsection{Distributed algorithms}

The algorithms of market heuristic automatically connect all the market players with interactions in a distributed manner. The algorithms of each agent are described as follows.

The yard agent iterates the following steps.

Step 1. Wait for the synchronous signal from the scheduler. If expired, terminate the algorithm.

Step 2. Generate a trading action upon three options: selling, buying, or keep silent using a uniform discrete distribution. If the trading action is to buy or keep silent, go to Step 5 .

Step 3. A vehicle is chosen to sell using Equation (1) or (2);

Step 4. Wait for the winning decision from the scheduler. If the signal of winning is notified, remove the vehicle from its vehicle set and go to Step 1.

Step 5. If there are any surplus vehicles left, insert them into its vehicle set and go to Step 1 .

A load agent iterates the following steps.

Step 1. Wait for the synchronous signal from the scheduler. If expired, terminate the algorithm.

Step 2. Generate a trading action in the same way as in the yard agent. If the trading action is to buy, go to Step 5; if the trading action is to sell, go to Step 3 .

Step 3. A vehicle is chosen to sell using Equation (1) or (2);

Step 4. Wait for the winning decision from the scheduler. If the signal of winning is notified, remove the vehicle from its vehicle set and go to Step 1;

Step 5. If this load agent is fully capacitated, go to
Step 9;

Step 6. Wait for the selling list from the scheduler;

Step 7. A vehicle is chosen to buy using Equation (3) or (4);

Step 8. Wait for the winning decision from the scheduler. If the signal of winning is notified, insert the vehicle into its vehicle set and go to Step 1.

Step 9. The decision about surplus trading is made by maximizing the benefit generated from trading, i.e., the difference between the deletion cost of one of the vehicles from the load agent and the insertion cost of one of the vehicles from the yard agent.

Step 10. Wait for the winning decision from the scheduler. If the signal of winning is notified, delete the vehicle selected for trading and insert the other vehicle from the yard agent into its vehicle set and go to Step 1.

The monitor agent iterates the following steps.

Step 1. Send synchronous signal to all the traders;

Step 2. Collect all the selling proposals;

Step 3. Send the list of selling proposals to the buying agents;

Step 4. Collect all the buying proposals;

Step 5. Decide the winning traders and notify them;

Step 6. If convergence criteria are satisfied, terminate the algorithm;

Step 7. Go to Step 1.

\subsection{Modeling shipment operations environment}

To validate the multi-agent distributed algorithm, we implemented the algorithm in a simulated shipment environment. Modeling real world operations is classified into two groups ${ }^{[14]}$ : one is analytical group, including mathematical model ${ }^{[15]}$, mixed integer programming ${ }^{[16,17]}$, and the other is simulation group, such as, system dynamics $^{[18,19]}$, agent-based modeling ${ }^{[20,21]}$, and discrete event simulation ${ }^{[22,23]}$. Analytical approach has been used for modeling and analyzing at strategic, high level, but due to their over-simplification and long computation time, they would be intractable for operational level of analysis. Simulation approach has been used for the 
Table 1. Performance comparison of four algorithms

\begin{tabular}{l|c|c|c|c}
\hline & EM & VRO & MST & MST Dyn \\
\hline Mean Distance (miles) & 79.22 & 80.52 & 81.80 & 79.65 \\
\hline $\begin{array}{l}\text { Mean Dwell Time } \\
\text { (days) }\end{array}$ & 3.00 & 2.88 & 2.77 & 1.84 \\
\hline Dwell Time Variance & 1.97 & 3.84 & 1.04 & 0.98 \\
\hline Truck Utilization & 0.98 & 0.98 & 0.98 & 1.00 \\
\hline
\end{tabular}

purpose of various supply chain modeling and analysis. The complexity of shipment yard business processes lends itself to select a simulation-based approach. In particular, considering the operational characteristics of shipment process, the discrete event simulation was the best fit to our objectives. A simulator was developed to emulate yard operations by visualizing all the processes and components. Simulation events include vehicle release from the plant, vehicle deployment to the yard, vehicle loading onto rail and truck, and vehicle shipment. Simulation entities include yard personnel, loading personnel, plant personnel, vehicles, railcars, and trucks. The simulator consists of four parts: (a) layout modeling of shipment yard; (b) operations modeling of shipment; (c) graphical animations of yard operations; and (d) planner for shipment algorithms. The market-based multi-agent decision algorithm was implemented within the simulator as part of the planner.

\section{Analysis Results}

A simulation was conducted to analyze the performance of the market-based dynamic optimization approach. Each simulation run produced different number of vehicles and, approximately, 200,000 vehicles were produced at an assembly plant during the 300 production days and shipped to 301 dealers having different demand percentages. As shown in Table 1, for each of all four algorithms, twenty simulation runs were conducted to reduce random effects and the simulation outputs were analyzed with the $5 \%$ significance. Simulation parameters, such as, production volume, shipment frequency, and number of yard operators were calibrated to ensure that the simulated current practice reflects actual current oper- ations. The vehicle deployment time to yard and the vehicle loading time on a truck are set to the exponential distribution with the mean of 10 minutes, reflecting real practice. Truck shipment is more important than rail shipment because of its higher shipment frequency, volume, and complexity. So we show the analysis results only for truck shipment.

Four algorithms, namely EM, VRO, MST, and MST Dyn (MST combined with dynamic optimization) are tested using the simulation. In particular, the MST Dyn algorithm considers the most recently updated vehicle and yard status. Table 1 summarizes the performance comparison for four algorithms. Regarding the mean travel distance, EM algorithm showed the best performance because it is conservative on the transportation cost. But for the dwell time, EM algorithm produced the worst performance because it is unrestrained on the dwell time and waits enough vehicles close to each other are released to the yard prior to building a new load. EM algorithm clusters vehicles only considering those vehicles in the same or neighboring destinations. Some vehicles could wait in the yard for a long time. MST Dyn algorithm produced the second best travel distance. For the mean dwell time, MST Dyn algorithm generated the best performance because it takes the dwell time requirement as the first priority and then, runs local optimization on transportation cost. MST algorithm follows next. VRO algorithm showed worse performance because it optimizes the transportation cost approximately without considering the dwell time requirement until the loads are all built. EM algorithm produced the worst performance that resulted in the maximum wait time limit on the contract between the assembly plant and the transportation carrier. For the variance of dwell time, MST Dyn produced the best performance and VRO algorithm the worst because the VRO only optimizes the travel distances without any consideration on the dwell time. Also notice that only MST Dyn produced $100 \%$ truck utilization. Based on these results, we can conclude that MST Dyn algorithm produced the best performance overall. 


\section{Managerial Implications}

Plant management has made an effort to reduce the OTD lead-time at manufacturing, for example, diminishing quality defects by tight quality assurance and control. Once a vehicle is complete in production and transferred to a transportation carrier, the ownership of the vehicle is moved to the carrier as well. The carrier has made much efforts to reduce the vehicle dwell time at shipment yard. In other words, two parties have done their best individually. However, the OTD dwell time and transportation cost cannot be improved unless these two parties are coordinated to one another. The dynamic optimization method facilitates the coordination of operations between the plant and the shipment yard because it continuously shares the updated changes with other agents.

In addition, the dynamic optimization could be enhanced by coordinating with the plant computer controlled manufacturing information systems, such as, manufacturing process monitoring and control, quality control, equipment management, and material management. These information systems could feed up-to-date production status and control data to the dynamic optimization algorithm for more accurate and timely decision making by capturing the most recent operational progress. By so doing, planning and executing the vehicle shipment is dynamically synchronized with computer controlled production throughput.

Plant management has been keen on those four measures analyzed in the previous section. Based on the insights of improved measures through dynamic optimization, the plant manager needs to establish follow-up actions to further enhance the measures by continuously improving associated business processes and encouraging active people involvement to support the dynamic decision making. As a follow-up for business process improvement, the plant manager could develop a new process to achieve timely reports of operational abnormality from plant and yard workers. People engagement could be fostered by developing training sessions for emphasis of the importance of supporting the OTD lead-time reduction effort.

The automaker's top management has also been very interested in the magnitude of the reduction in the OTD lead-time along with the transportation cost because the resulting rippling effect would apply to other assembly plants and distribution centers. The rippling effect would be additionally magnified when production volume increases.

\section{Conclusions and Future Study}

Our analysis results have showed that dynamic decision algorithm based on the multi-agent computational architecture and market-based heuristics improved the performance of vehicle shipment. It could significantly improve customer satisfaction by reducing mean and variance of dwell time, save transportation cost by reducing travel distance, and decrease labor cost by increasing labor utilization. The results have demonstrated that the dynamic optimization approach produced better performance than any other static algorithms that are limited in capturing the dynamics of the shipment business environment. The dynamic optimization could further assist the automaker to reduce warranty cost by identifying defective (abnormal) vehicles prior to shipment to customers. Another important goal in managing a shipment yard is to determine the right number of people. Excessive operational costs usually arise when there is an oversupply or under-supply of labor ${ }^{[17]}$.

Moreover, when more accurate and timely vehicle information is available to agents, the dynamic optimization method could accomplish further reduction of the OTD lead-time and the transportation cost. This may require establishing information infrastructure that cultivates seamless business connectivity between existing plant manufacturing systems and vehicle delivery systems.

As a further study, we need to conduct a comprehensive research on more advanced algorithms that could be successfully applied for dynamic optimization. This may include the algorithms based on immunization, market behaviors or insect world, or distributed metaheuristic algorithms, such as genetic algorithm, simulated 
annealing, and tabu search. An effort is needed to provide guidance on which algorithm produces best performance under real-time environment.

\section{References}

1. Costy, T., Truss, L., and Tew, J., "Integrated supply chain management exploratory project mid-term report", GM Research Report ESL-44, 2000.

2. Cormen, T., Leiserson, C., Rivest, R., and Stein, C., Introduction to Algorithms (Second Edition). The MIT Press, 2001.

3. Kim, J. D., Tang, K., Kumara, S., Yee, S. T., and Tew, J., "Value analysis of location-enabled radio frequency identification information on delivery chain performance", International Journal of Production Economics, Vol. 112, No. 1, pp. 403-415, 2007.

4. Schieber, B., Bar-Noy, A., Bhatia, R., and Naor, J., "Minimizing service and operation costs of periodic scheduling”, Math. Oper. Res., Vol. 27, pp. 518-544, 2002.

5. Vaserstein, L. N. and Byrne, C. C., Introduction To Linear Programming. Prentice Hall/Pearson Education, 2003.

6. Weiss, G., Multiagent Systems - A Modern Approach to Distributed Artificial Intelligence. The MIT Press, Cambridge, Massachusetts; London, England, 1999.

7. Wooldridge, M. J., An Introduction to Multi-Agent Systems. Chichester, UK: Wiley, 2002.

8. Hong, Y., Chen, G., and Bushnell, L., "Distributed observers design for leader-following control of multi-agent networks", Automatica, Vol. 44, No. 3, pp. 846-850, 2008.

9. Nedic, A. and Ozdaglar, A., "Distributed subgradient methods for multi-agent optimization", IEEE Transactions on Automatic Control, Vol. 54, No. 1, pp.48-61, 2009.

10. Nedic, A., Ozdaglar, A., and Parrilo, P.A., "Constrained consensus and optimization in multi-agent networks", IEEE Transactions on Automatic Control, Vol. 55, No. 4, pp. 922-938, 2010.

11. Davidson, P., Johansson, S. J., Persson, J. A., and Wernstedt, F., "Agent-based approaches and classical optimization techniques for dynamic distributed resource allocation: a preliminary study", Working paper, Department of Software Engineering and Computer Science Blekinge Institute of Technology, Soft Center, 37225 Ronneby, Sweden, 2003.
12. Tang, K. and Kumara, S., "Double auction market mechanism: a distributed negotiation protocol to model an e-procurement problem", The proceedings of IEEE Automation Science and Engineering, 2004.

13. Oh, S. C., Yee, S.T., and Kim, T. W., “Agent-based shipment algorithm for capacitated vehicle routing problem with load balancing", Journal of the Korean Institute of Industrial Engineers, Vol. 32, No. 3, 200-209, 2006.

14. Huang, G. Q., Lau, J. S. K., and Mak, K. L., "The impacts of sharing production information on supply chain dynamics: a review of the literature", International. Journal of. Production Research, Vol. 410, No. 7, pp. 1483-1517, 2003.

15. Lee, H. L., So, K. C., and Tang, C. S., "The value of information sharing in a two-level supply chain", Management Science, Vol. 46, pp. 626-643, 2000.

16. Sterman, J. D., Business Dynamics: System Thinking and Modeling for a Complex World (Boston, MA: McGraw-Hill), 2000.

17. Anderson, B. M., Gremban, K. D., and Young, B. A., "Shipyard operational improvement through process management", Ship Production Symposium, 1997.

18. Anderson, E. G., Fine, C. H., and Deployer, G. G., "Upstream volatility in the supply chain. the machine tool industry as a case study", Production and Operations Management, 9, pp. 239-261, 2000.

19. De Souza, R., Song, Z. C., Liu, C. Y., "Supply chain dynamics and optimization", Integrated Manufacturing Systems, Vol. 11, pp. 348-364, 2000.

20. Brandolese, A., Brun, A., and Portioli-Straudacher, A., "A multi-agent approach for the capacity allocation problem", International Journal of Production Economics, Vol. 66, pp. 269-285, 2000.

21. Gjerdrum, J., Shah, N., and Papageorgiou, L. G., "A combined optimization and agentbased approach to supply chain modeling and performance assessment", Production Planning and Control, Vol. 12, No. 1, pp. 81-88, 2001.

22. Beamon, B. M. and Chen, V. C. P., "Performance analysis of conjoined supply chains", International Journal of Production Research, Vol. 39, pp. 3195-3218, 2001.

23. Banerjee, S., Banerjee, A., Burton, J., and Bistline, W., "Controlled partial shipments in two-echelon supply chain networks: a simulation study", International Journal of Production Economics, Vol. 71, pp. 91-100, 2001. 


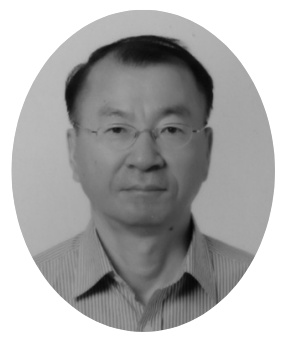

John Yee (styee@hotmail.com)

1998 Ph.D., Industrial \& Manufacturing Engineering, Penn. State Univ.

$2013 \sim$ Present US Department of the Treasury

2014 Present Professor at SungKyunKwan Univ.

Research Interests : Applied Operations Research, Technology Integration and Management, Business Analytics 